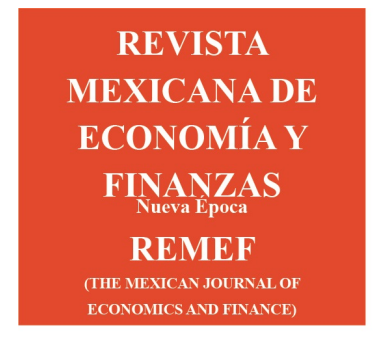

\begin{tabular}{c} 
Revista Mexicana de Economía y Finanzas \\
Nueva Época \\
Volumen 15 Segundo Número Especial Aniversario \\
Agosto $2020 \quad$ pp. $537-549$ \\
DOI: https ://doi.org/10.21919/remef.v15i0.545 \\
(Recibido: $13 /$ abril/2020, aceptado: $17 /$ agosto/2020) \\
\hline
\end{tabular}

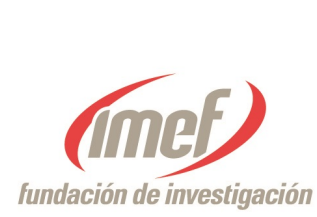

SNEA - Entrepreneurial research in emerging economies: recent advances Editor Invitado: Dr. Samuel Mongrut

\title{
Inclusión financiera y productividad: el caso colombiano
}

\author{
Rafael Gómez Gómez ${ }^{1}$ \\ Universidad de Manizales, Colombia \\ Eliana Morales Zuluaga ${ }^{2}$ \\ Universidad de Manizales, Colombia \\ Juan Felipe Castellanos Martínez ${ }^{3}$ \\ Universidad de Manizales, Colombia
}

\section{Resumen}

El presente trabajo pretende identificar los efectos de la inclusión financiera sobre la productividad en Colombia. Para su medición se utiliza una muestra de datos de tipo panel de efectos fijos tomando la Encuesta Anual Manufacturera. La variable dependiente corresponde a la productividad a nivel de los establecimientos, en función de un portafolio de productos financieros. Los resultados indican que los esfuerzos de inclusión entendidos como la adopción servicios financieros no implican una mayor productividad. Por lo tanto, muestran fallos persistentes que limitan el acceso al capital y a los factores productivos. Es recomendable para futuros análisis incorporar los instrumentos de ahorro empleados por las unidades productivas. Este estudio abre entonces un importante debate en el caso colombiano y en economías similares, pues diversos estudios sectoriales, esfuerzos gremiales y la política pública se enfocan en la adopción de un portafolio de productos que instrumentaliza la inclusión sin lograr los beneficios que se pretenden. Finalmente, se concluye de este estudio que el acceso a una diversidad de servicios financieros no garantiza el aumento de la productividad y que incluso puede deteriorarla.

Clasificación JEL: G1, G22, O14, L25

Palabras clave: inclusión financiera, seguros, industria manufacturera, finanzas para pymes

\section{Financial inclusion and productivity: the Colombian case}

\section{Abstract}

This paper aims to identify the effects of financial inclusion on productivity in Colombia. For its measurement we use a fixed effects panel data sample from the Annual Manufacturing Survey. The dependent variable corresponds to productivity at the establishment level, as a function of a portfolio of financial products. The results indicate that inclusion efforts understood as the adoption of financial services do not imply higher productivity. Therefore, they show persistent failures that limit access to capital and productive factors. It is advisable for future analyses to incorporate the savings instruments used by productive units. This study thus opens an important debate in the Colombian case and in similar economies, since various sectoral studies, trade union efforts and public policy focus on the adoption of a portfolio of products that instrumentalizes inclusion without achieving the intended benefits. Finally, this study concludes that access to a diversity of

\footnotetext{
${ }^{1}$ Email: rgomez@umanizales.edu.co

${ }^{2}$ Email: emorales@umanizales.edu.co

${ }^{3}$ Email: jcastellanos@umanizales.edu.co

*Sin fuente de financiamiento para el desarrollo de la investigación
} 


\section{Abstract}

financial services does not guarantee an increase in productivity and may even deteriorate it. JEL Classification: G1, G22, 014, L25

Keywords: financial inclusion, insurance, manufacturing industry, finance for SME, SME

\section{Introducción}

Según Isenberg $(2010 ; 2011)$ la financiación es uno de los principales retos para implantar un modelo de creación de empresas, así como su crecimiento en un territorio determinado. En la misma línea Acemoglu y Robinson (2012) aseveran que el camino para el desarrollo financiero es "Tener una idea, crear una empresa y conseguir un préstamo" (p. 48).

El contexto mundial demuestra como el acceso a diversas fuentes de financiación y en si misma la inclusión financiera permiten transitar hacia un incremento en la productividad de los factores a nivel empresarial (Sarma \& Pais, 2010; Khyareh, Khairandish \& Torabi, 2018). En dicho marco se pretende desarrollar el presente trabajo, ilustrando en primera medida como se ha conceptualizado la inclusión financiera, que esfuerzos se han documentado y por último cuantificar el efecto de la inclusión, como un portafolio de productos financieros en relación con la productividad.

Un punto de partida relevante es la caracterización de un fenómeno que en Latinoamérica (Fuenzalida y Mongrut, 2010) al igual que varios países emergentes, han presentado un rezago en términos de acceso al capital y completitud de mercados. Condición que también es destacada por Manuelito y Jiménez (2010), al denotar una restricción estructural que limita los flujos de ahorro a inversión y encarece los costos transaccionales.

Restricción que a su vez limita la posibilidad de surgimiento y crecimiento empresarial, como consecuencia de exclusión financiera. Allen y otros (2012) presentan de manera problemática las consecuencias de la exclusión financiera y la describen como un fenómeno donde las empresas tienen que financiarse con recursos propios, característica que se manifiesta en ocasiones como trampas de pobreza o como la directa inhibición de la productividad.

Algunos trabajos como el de Sharma (2016) destacan en una economía emergente como la India, un efecto positivo así como una causalidad directa de la inclusión financiera en el crecimiento económico. Al igual que trabajos como los de Kessler y otros (2017) en el contexto de Sudáfrica.

Sin embargo, existen estudios que plantean una importante divergencia como es el caso de Kim, Yu y Hassan (2018) que establece un efecto positivo de la inclusión, pero estima que la relación de causalidad es mutua con respecto al crecimiento económico. En contraposición con diversos estudios, que sitúan la inclusión como una herramienta de crecimiento en la productividad.

En una línea de evidencias similares, Maldonado y Tejerina (2010) presentan algunos esfuerzos gubernamentales, como algunos programas de ayuda social y la forma como han incorporado dentro de sus políticas la vinculación de los beneficiarios en el sistema financiero (Karpowicz, 2016). Mas la evidencia demuestra que la vinculación no es suficiente, pues los altos costos transaccionales resultan en una persistente restricción de acceso al crédito.

Thoene y Turriago-Hoyos (2017) presentan una revisión sobre el caso colombiano, en el cual demarcan los efectos negativos de la exclusión financiera siendo una limitante para el mejoramiento en las condiciones empresariales y la calidad de vida de los ciudadanos.

Algunas vertientes destacan factores que limitan la inclusión como son las brechas de género, la escolaridad de los propietarios y fenómenos de localización. Así como evidencian problemas de concentración bancaria. 
que en ultimas desvirtúan el proceso de incorporación al sistema financiero pues se presentan restricciones en el canal de crédito (Chauvet \& Jacolin, 2017).

En general, se plantea un panorama de múltiples capas pues la inclusión entendida como la adopción de un portafolio de servicios financieros requiere de condiciones de entorno y estructura social. Frente a lo anterior es persistente la pregunta ¿si hasta el momento las medidas de inclusión adoptadas en Colombia tienen efectos positivos en términos de productividad y crecimiento empresarial?

Para responder la anterior pregunta, el presente trabajo pretende reconstruir las condiciones de inclusión financiera en Colombia para empresas en diferentes etapas de evolución según la Encuesta Anual Manufacturera (Dane, 2018), como una visión de la capacidad de incrementar la productividad en el contexto industrial. Para tal fin, se calculan modelos de regresión con diferentes ajustes, para establecer la magnitud y la dirección de la relación entre la inclusión financiera y la productividad.

El principal impacto se centra en comprender el efecto de la adopción de un portafolio de servicios y su reflejo en términos de productividad, lo anterior en línea con los esfuerzos adoptados desde la política pública y el sector financiero hacia inclusión.

\section{Marco teórico}

La inclusión financiera se ha convertido en una pieza clave para mejorar el desarrollo económico (Ouma, Odongo y Were, 2017), reducir barreras a la hora de acceder a los servicios financieros (Burns, 2018), aumentar la productividad (Zanzzi, Bonilla \& Gaibor, 2015), disminuir la asimetría de la información (Fan \& Zhang, 2017), la mitigación de la pobreza (Cobb, Wry \& Zhao, 2016) y un aumento del bienestar (Forcadell \& Aracil, 2017).

Para Ajide (2020), Goel y Madan (2019) y Drori y otros (2018) la inclusión financiera es el medio para fomentar la creación y crecimiento empresarial, debido a diferentes escenarios en los que se enfrentan las pequeñas y medianas empresas para acceder a productos financieros, teniendo en cuenta las restricciones (Di Giannatale y Roa, 2016) y los costos (Granda, Hamann y Tamayo, 2019). Sin embargo, cuando logran confluir estas dos fuerzas (Gimet y Lagoarde, 2012) se incrementa la actividad empresarial (Newman, Schwarz y Ahlstrom, 2017), la inversión en nuevos proyectos (Acosta y otros, 2018) y mejora el desempeño siempre y cuando se aborde de manera holística y competitiva (Jaksic y Jaksic, 2018).

De igual forma las instituciones financieras deben mantenerse en transformación permanente aumentando así la competitividad, y así la eficiencia, un ejemplo es el desarrollo de desarrollo de plataformas digitales que aumenten la cobertura del sistema (Zins \& Weill, 2016), además de fortalecer la educación financiera como principal medio para alcanzar la inclusión (Kurowski \& Laskowska, 2016) a través de la formación en las diferentes alternativas de financiación, costos y garantías (Goswami, Hazarika y Handique, 2017) para la estimulación de la productividad empresarial (Bialek \& Gabryelczyk, 2015) evitando pérdidas friccionales en el sistema de crédito (Kimmitt y Muñoz, 2017).

La disponibilidad en las fuentes de financiación, la disminución en los requisitos de capital y la facilidad en el acceso muestran evidencias prometedoras para el aumento en la productividad, la creación y aceleración de empresas (Ho \& Wong, 2007). Resaltando el papel protagónico que posee el acceso a financiamiento, inversión y productos de aseguramiento (Cumming y otros, 2019) en una relación positiva con la productividad (Bhaird, Robyn \& Freel, 2019).

Pese a lo anterior, persisten retos para que la economía incorpore plenamente los beneficios de un sector financiero desarrollado, Sarma y Pais (2010) desagregan algunos de los factores que intervienen en la inclusión, como: el capital humano, infraestructura y conectividad. Así como revelan una relación inversamente significativa con el coeficiente de Gini. Denotando el efecto retardante que la concentración del ingreso tiene 
sobre la inclusión financiera.

Otro hallazgo relevante se centra en el tamaño de los bancos (Sarma \& Pais, 2010) pues presentan evidencias frente a niveles de capitalización relativamente altos y una marcada tendencia a un menor esfuerzo en términos de inclusión.

En línea con lo anterior, Nikaido, Pais y Sarma (2015) discuten evidencias en países emergentes que cuestionan la inclusión desde una perspectiva más amplia y surgen de nuevo categorías como el nivel de escolaridad de los empresarios y sus directivos, brechas de género y la localización geográfica (Zins \& Weill, 2016) presentando algunas variables exógenas que dificultan el proceso de inclusión.

Aunque el marco restrictivo no es exclusivamente exógeno (Anzoategui, Demirgüç-Kunt, \& Martínez, 2014; Kostov, Arun \& Annim, 2015) demuestra que la simple adopción de un portafolio de servicios financieros no se traduce verticalmente en una mayor inclusión, pues las brechas de acceso a financiación permanecen latentes. Incluso una un evidente fallo de mercado es el expuesto por Chauvet y Jacolin (2017), en la cual concluyen que países con alta concentración en el mercado de crédito, problemas de gobernanza en sus instituciones y brechas de información relativamente altas, no logran transmitir el beneficio del desarrollo financiero al sector productivo pese a la sofisticación de sus productos.

En conclusión, si bien la inclusión financiera presenta beneficios en la productividad dentro de la literatura, también son identificables diversas barreras y complejidades que no permiten la plena expresión de los canales de créditos y por ende de la productividad de los factores.

\section{Metodología}

Con el fin de estimar la incidencia de la inclusión financiera sobre la productividad se utilizaron los microdatos de la Encuesta Anual Manufacturera (EAM), elaborada por el Departamento Administrativo Nacional de Estadística (Dane, 2018). La información sobre este instrumento es publicada anualmente por el Dane. La información más reciente de esta encuesta corresponde a la versión de 2018, con la cual se construyeron los análisis de este documento; por lo tanto, la base de datos utilizada cuenta con 40.682 observaciones correspondientes a establecimientos industriales para cinco años; 2014, 2015, 2016, 2017 y 2018. El carácter de dicha encuesta es censal, e incluye todos los establecimientos industriales colombianos diferentes de micro establecimientos.

Para calcular la incidencia de la inclusión financiera sobre la productividad industrial se planteó un modelo de regresión de datos panel (ecuaciones 4 y 5), donde la variable dependiente fue construida a través de una regresión semiparamétrica, siguiendo el método planteado por Levinsohn \& Petrin (2003).

Partiendo de la función de producción con un multiplicador de cambio técnico (Solow, 1957)

$$
Y=A(t) f(K, L)
$$

Donde $Y$ representa la producción, $K$ representa el capital utilizado en producción y $L$ representa la cantidad de factor trabajo y $A(t)$ puede interpretarse como el factor de cambio técnico. En términos generales se asume que dicha ecuación sigue una forma funcional Cobb-Douglas de la siguiente manera

$$
Y_{i t}=A_{i t} K_{i t}^{\beta k} L_{i t}^{\beta l}
$$

Donde $i$ corresponde a cada una de las unidades productivas evaluadas. Por su parte se incorporar las elasticidades parciales con respectivamente para capital y trabajo $\beta_{k}$ y $\beta_{l}$. 
La función tipo Cobb-Douglas (2) anteriormente descrita puede ser expresada en forma lineal

$$
y_{i t}=\beta_{0}+\beta_{k} k_{i t}+\beta_{l} l_{i t}+\varepsilon_{i t}
$$

Donde el uso de las letras en su forma minúscula posee una connotación logarítmica, condición que conlleva a interpretar

$$
\ln \left(A_{i t}\right)=\beta_{0}+\varepsilon_{i t}
$$

Donde $\beta_{0}$ es una medida de la eficiencia media de las empresas en una ventana de observación, y $\varepsilon_{i t}$ corresponde a la desviación frente a la media de cada unidad productiva en el tiempo. Van Beveren (2007) desdobla el error en dos términos, el primero es un componente observable que permite obtener la productividad de los factores de manera empírica ecuación (5)

$$
\hat{\omega}_{i t}=y_{i t}-\hat{\beta}_{k} k_{i t}-\hat{\beta}_{l} l_{i t}
$$

Y un componente inobservable $u_{i t}^{q}$ corresponde a un error de medición.

Para la estimación se utiliza el método planteado por Levinsohn \& Petrin (2003) modificando la ecuación (3) resultando

$$
y_{i t}=\beta_{0}+\beta_{k} k_{i t}+\beta_{\iota} \iota_{i t}+\beta_{l} l_{i t}+\omega_{i t}+u_{i t}^{q}
$$

Donde t representa bienes intermedios utilizados en la producción la cual esta en función de productividad y capital como se describe en la ecuación 7

$$
\iota_{i t}=f_{t}\left(\omega_{i t}, k_{i t}\right)
$$

Las variables $\omega_{i t}, k_{i t}$ son monótonas y por ende pueden ser invertidas. Posteriormente reemplazada en la ecuación (6) para obtener así la

$$
y_{i t}=\beta_{0}+\beta_{k} k_{i t}+\beta_{l} l_{i t}+f_{t}^{-1}\left(k_{i t}, \iota_{i t}\right)+u_{i t}^{q}
$$

$f_{t}^{-1}$ tiene un tratamiento no paramétrico (Olley y Pakes, 1996), por lo tanto, la estimación de $\beta_{0}$ así como $\beta_{k}$ se obtiene mediante un termino compuesto $\phi_{i t}\left(\iota_{i t}, k_{i t}\right)$, de donde se deduce

$$
y_{i t}=\beta_{l} l_{i t}+\phi_{i t}\left(\iota_{i t}, k_{i t}\right),+u_{i t}^{q}
$$

En un segundo momento se procede a estimar

$$
y_{i t}^{*}=\beta_{0}+\beta_{k} k_{i t}+\beta_{l} l_{i t}+E\left[\omega_{i t} \mid \omega_{i t-1}\right]+u_{i t}^{q *}
$$

Donde $y_{i t}^{*}$ es la producción neta de la contribución del trabajo, y $u_{i t}^{q *}$ la sumatoria de $u_{i t}^{q} y$ las expectativas de innovación en el periodo anterior.

Posteriormente se procede a estandarizar las productividades con media cero y desviación igual a uno, para cada grupo industrial (CIIU a dos dígitos) mediante la siguiente ecuación

$$
Z_{i j}=\frac{P T F_{i j}-P \bar{T} F_{j}}{s_{j}}
$$

Donde $Z_{i j}$ es el puntaje estandarizado de la productividad total factorial de la empresa i que pertenece al grupo industrial j. 
$P T F_{i j}$ es el logaritmo de la productividad de la empresa $i$ que pertenece al grupo industrial $j$.

$P \bar{T} F_{j}$ es el promedio del logaritmo productividad del grupo industrial $j$ en su forma.

$s_{j}$ es la desviación estándar del logaritmo de la productividad total factorial de las empresas pertenecientes al grupo $j$

En cuanto a las variables de inclusión financiera, se tomó el valor total del leasing financiero $(L)$, el valor total de los seguros $(S)$ y el valor total de los intereses causados sobre los préstamos como un proxy del endeudamiento $(E)$. Desde este enfoque puede existir una limitación, ya que una correlación entre las variables de inclusión financiera y la productividad puede estar sobreestimada debido al efecto del tamaño de las empresas. Así, estas variables se llevaron a niveles comparables dividiendo los valores obtenidos entre la producción total $(Y)$; de la misma forma, se evaluó el modelo dividiendo los valores obtenidos entre el valor total de las ventas $(V)$.

De esta manera, el modelo planteado tiene la forma descrita en las ecuaciones 2 y 3.

$$
\begin{aligned}
& A_{i t}=\beta_{0}+\beta_{1} \frac{L_{i t}}{V_{i t}}+\beta_{2} \frac{S_{i t}}{V_{i t}}+\beta_{3} \frac{E_{i t}}{V_{i t}}+u_{i t} \\
& A_{i t}=\beta_{0}+\beta_{1} \frac{L_{i t}}{Y_{i t}}+\beta_{2} \frac{S_{i t}}{Y_{i t}}+\beta_{3} \frac{E_{i t}}{Y_{i t}}+u_{i t}
\end{aligned}
$$

El análisis de estos modelos llevó a evaluar la conveniencia de utilizar las variables del modelo en su forma logarítmica. Los modelos evaluados con esta forma funcional se muestran en las ecuaciones 4 y 5 .

$$
\begin{aligned}
& \log \left(A_{i t}\right)=\beta_{0}+\beta_{1} \log \left(\frac{L_{i t}}{V_{i t}}\right)+\beta_{2} \log \left(\frac{S_{i t}}{V_{i t}}\right)+\beta_{3} \log \left(\frac{E_{i t}}{V_{i t}}\right)+u_{i t} \\
& \log \left(A_{i t}\right)=\beta_{0}+\beta_{1} \log \left(\frac{L_{i t}}{Y_{i t}}\right)+\beta_{2} \log \left(\frac{S_{i t}}{Y_{i t}}\right)+\beta_{3} \log \left(\frac{E_{i t}}{Y_{i t}}\right)+u_{i t}
\end{aligned}
$$

Para determinar el uso de efectos fijos o efectos aleatorios, se calculó la prueba de Hausman. Debido a que todas las variables utilizadas se encontraron en pesos colombianos, se deflactaron llevándolas a valores de 2018. Para las variables de valor agregado, capital y consumo intermedio se utilizó el índice de producción real de la industria manufacturera colombiana con enfoque territorial, el cual permite desagregación a nivel de tres dígitos del CIIU. El resto de las variables se deflactaron utilizando el índice de precios al consumidor. Tanto los análisis efectuados como el ajuste de los modelos de datos panel fueron realizados por medio del software Stata 11.

\section{Modelo de contrastación}

Tras evaluar la significancia de los coeficientes obtenidos en los modelos de regresión, y clasificarla se procede a realizar una observación sobre los valores que puede tomar $\beta$ :

Si $\beta$ presenta un coeficiente positivo cuyo valor sea mayor que 0 , presentan un a porte positivo en términos de productividad, implicando que el uso de alguno de los instrumentos contemplados en la medición de inclusión aporta de manera positiva a la productividad.

Un coeficiente igual a 0 implican neutralidad del uso del instrumento de inclusión, denotando que la inclusión de dicho producto o servicio, no genera ningún aporte a la productividad

$\mathrm{Y}$ un coeficiente menor que 0 implican una pérdida de productividad, denotando un impacto negativo a raíz de la adquisición o uso de un producto o servicio financiero definido en inclusión. 
Los efectos captados a través de la regresión entonces permitirán observar en términos de resultado, los aportes, la neutralidad o la inhibición que puede estar representando el portafolio de productos financiero sobre la productividad de los factores en pequeñas y medianas empresas para Colombia.

\section{Resultados}

La tabla 1 resume las estadísticas descriptivas para las variables relevantes dentro de los modelos propuestos. Inicialmente, se observa que no hay una incidencia relevante de valores faltantes y que todas las variables propuestas reflejan la heterogeneidad de los establecimientos industriales. Esto se puede apreciar en los valores especialmente altos que se obtienen para la desviación estándar en comparación con los promedios. Una cuestión problemática encontrada en la exploración inicial de los datos es la alta incidencia de valores iguales a cero. Para las variables de seguros y productividad, la existencia de ceros no es un problema; pero, de acuerdo con los datos utilizados, para 10.706 establecimientos el total de los intereses causados sobre los préstamos es igual a cero. Lo mismo ocurre en 37.937 establecimientos para la variable valor total del leasing.

Tabla 1. Estadísticas descriptivas de las variables utilizadas

\begin{tabular}{|c|c|c|c|}
\hline Variable & N & Promedio & Desviación estándar \\
\hline Productividad & 39,769 & 18.58 & 36.93 \\
\hline Leasing & 40,689 & $45,828.61$ & 444,237 \\
\hline Endeudamiento & 40,692 & $314,927.2$ & $4,475,758$ \\
\hline Seguros & 40,692 & $55,296.65$ & $880,621.1$ \\
\hline
\end{tabular}

Fuente: cálculos propios, a partir de la Encuesta Anual Manufacturera (Dane, 2014-2018).

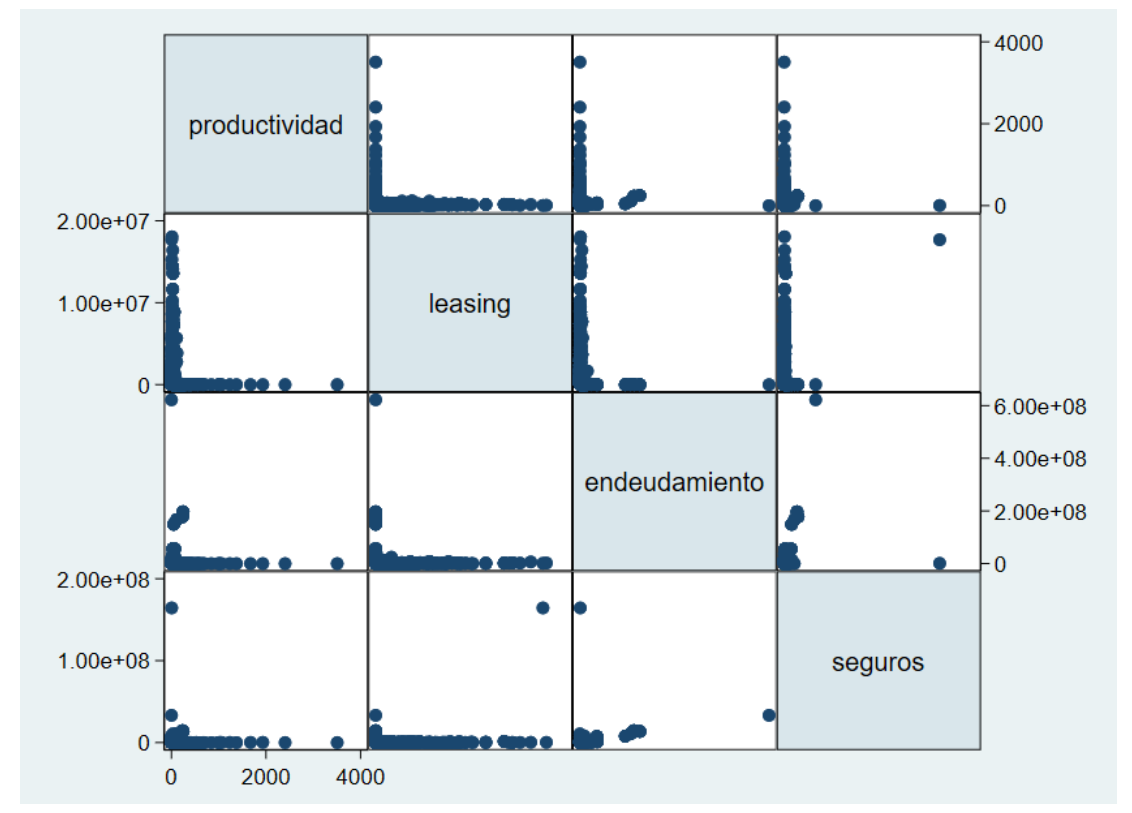

Grafico 1. Diagramas de dispersión de la productividad y las variables de la inclusión financiera

Fuente: elaboración propia con base en la encuesta Anual Manufacturera (Dane, 2014-2018).

El gráfico 1 muestra que, de existir alguna relación entre estas variables, esta no corresponde a una forma funcional lineal. El gráfico sugiere relaciones de tipo logarítmica, principalmente entre las variables explicativas y la variable dependiente. De esta manera, los coeficientes de correlación son bajos, como lo sugiere la tabla 2 . 
Tabla 2. Correlaciones entre la productividad y las variables de la inclusión financiera

\begin{tabular}{|c|c|c|c|c|}
\hline & Productividad & Leasing & Endeudamiento & Seguros \\
\hline Productividad & 1.00 & & & \\
\hline Leasing & 0.01 & 1.00 & & \\
\hline Endeudamiento & 0.06 & 0.02 & 1.00 & \\
\hline Seguros & 0.04 & 0.19 & 0.32 & 1.00 \\
\hline
\end{tabular}

Fuente: elaboración propia, con base en la encuesta Anual Manufacturera (Dane, 2014-2018).

A pesar de que la forma logarítmica mejora sustancialmente las correlaciones mostradas, la alta incidencia de valores cero en las variables de endeudamiento y leasing hace que con esta transformación se pierda una parte importante de las observaciones.

Inicialmente se evalúan tres modelos, en todos los cuales la variable dependiente corresponde a la productividad total de los factores (tabla 3). En estos, se hace un tratamiento de los datos como si correspondieran a corte transversal. El modelo 1 toma como variables independientes las tres variables de inclusión financiera en su nivel habitual, el modelo 2 divide estas variables entre las ventas y el modelo 3 las divide entre los seguros. Los coeficientes fueron estadísticamente significativos en la mayor parte de los casos. La dirección de los coeficientes no es consistente en estos modelos iniciales, pero en estos aún no se considera la corrección de la forma funcional ni la estructura de datos panel.

Tabla 3. Resultados del modelo de regresión

\begin{tabular}{|l|c|c|c|c|c|c|}
\hline \multirow{2}{*}{ Seguros } & \multicolumn{2}{|c|}{ Modelo 1 } & \multicolumn{2}{c|}{ Modelo 2 } & \multicolumn{2}{c|}{ Modelo 3 } \\
\hline \multirow{2}{*}{ Endeudamiento } & $6.48 \mathrm{e}-07$ & $* * *$ & & & & \\
\hline \multirow{2}{*}{ Leasing } & $(2.24 \mathrm{e}-07)$ & & & & & \\
\hline & $4.61 \mathrm{e}-07$ & $* * *$ & & & & \\
\hline \multirow{2}{*}{ Seguros/ventas } & $(4.31 \mathrm{e}-08)$ & & & & & \\
\hline \multirow{2}{*}{ Endeudamiento/ventas } & $8.63 \mathrm{e}-07$ & $* *$ & & & & \\
\hline & $(4.21 \mathrm{e}-07)$ & & & & & \\
\hline \multirow{2}{*}{ Leasing/ventas } & & & -0.67 & & & \\
\hline \multirow{2}{*}{ Seguros/producción } & & & $(1)$ & & & \\
\hline \multirow{2}{*}{ Endeudamiento/producción } & & & -0.19 & & & \\
\hline & & & $(0.17)$ & & & \\
\hline & & & -5 & $* * *$ & & \\
\hline \multirow{2}{*}{ Leasing/producción } & & & & & -4.87 & $* *$ \\
\hline \multirow{2}{*}{ constante } & & & & & $(2.43)$ & \\
\hline R cuadrado & 18.36 & $* * *$ & 18.58 & $* * *$ & 18.64 & $* * *$ \\
\hline $\mathrm{N}$ & $(0.19)$ & & $(0.18)$ & & $(0.18)$ & \\
\hline
\end{tabular}

Errores estándar en paréntesis. Significancia de los coeficientes: ${ }^{* * *}=\mathrm{p}<0,01,{ }^{* *}=\mathrm{p}<0,05,{ }^{*}=\mathrm{p}<0,1$

Fuente: elaboración propia, con base en la encuesta Anual Manufacturera (Dane, 2014-2018).

De esta forma se calculan nuevos ajustes que incluyen las formas logarítmicas sugeridas por el análisis 
exploratorio. Como se mencionó previamente, el número de observaciones se reduce en una amplia magnitud debido a la incidencia de valores cero en algunas de las variables. Además de modificar la forma funcional, se utilizan métodos de datos panel, después de que la regresión de efectos fijos fue validada a través del test de Hausman. Con este ajuste, la relación entre las variables de inclusión financiera y la productividad es inversa, de manera consistente en ambos modelos (tabla 4).

Tabla 4. Resultados del modelo de regresión con ajuste logarítmico

\begin{tabular}{|l|c|c|c|c|}
\hline \multirow{2}{*}{ Log seguros/ventas } & \multicolumn{2}{|c|}{ Modelo 4 } & \multicolumn{2}{c|}{ Modelo 5 } \\
\hline \multirow{2}{*}{ Log endeudamiento/ventas } & -0.13 & $* * *$ & & \\
\hline & $(0.02)$ & & & \\
\hline \multirow{2}{*}{ Log leasing/ventas } & -0.10 & $* * *$ & & \\
\hline & $(0.02)$ & & & \\
\hline \multirow{2}{*}{ Log seguros/producción } & -0.01 & $*$ & & \\
\hline & $(0.01)$ & & & \\
\hline \multirow{2}{*}{ Log endeudamiento/producción } & & & -0.16 & $* * *$ \\
\hline & & & $(0.02)$ & \\
\hline \multirow{2}{*}{ Log leasing/producción } & & & -0.12 & $* * *$ \\
\hline \multirow{2}{*}{ Constante } & & & -0.02 & $* *$ \\
\hline & & & $(0.01)$ & \\
\hline Sigma u & -0.93 & $* * *$ & -1.19 & $* * *$ \\
\hline & $(0.16)$ & & $(0.16)$ & \\
\hline Sigma e & 0.66 & & 0.65 & \\
\hline N & 0.38 & & 0.37 & \\
\hline en paréntesis. Significancia de los coeficientes: *** & & & \\
\hline
\end{tabular}

Errores estándar en paréntesis. Significancia de los coeficientes: ${ }^{* * *}=\mathrm{p}<0.01,{ }^{* *}=\mathrm{p}<0.05,{ }^{*}=\mathrm{p}$
Fuente: elaboración propia, con base en la encuesta Anual Manufacturera (Dane, 2014-2018).

\section{Discusión de los resultados}

$\mathrm{Al}$ evaluar los valores obtenidos del modelo se puede determinar un efecto negativo en el nivel de acceso a diversos productos financieros. Una posible condición que podría esbozar unos coeficientes negativos en orden con las barreras planteadas por Chauvet \& Jacolin, (2017). Las cuales radican en la concentración excesiva de mercado y en las evidencias de colusión (Delvasto 2006) (Tovar, Jaramillo y Hernández, 2007) que se han documentado sobre el caso colombiano.

El fenómeno de colusión se traduce en ineficiencia descrita por Ferrari (2009), la cual plantea los retos de una inclusión financiera en una economía emergente, donde los bancos dominantes ejercen una captura de la renta en un evidente fallo de mercado. Trasladando así valor desde los sectores productivos hacia los sectores de servicios financieros.

Otra vertiente, que puede ilustrar los resultados negativos en términos de la productividad, puede estar relacionada con el fenómeno de captura del ahorro por parte de las emisiones de deuda de los gobiernos latinoamericanos, que limita el tránsito entre ahorro e inversión (Manuelito y Jiménez, 2010; 2013). De esta manera puede generarse un efecto de mayor demanda de dinero que, a su vez, presiona las tasas de interés al alza, y restringe la productividad de aquellas empresas que, si bien acceden a endeudamiento, están sometidas a pagar tasas de interés distorsionadas por una restricción de crédito (Stiglitz \& Weiss, 1981). 
Una tercera visión, que puede dilucidar una arista adicional al problema, consiste en el nivel de formación financiera y la idoneidad que poseen los agentes económicos que trabajan en las empresas, tal como lo expone Rojas (2016) similar a los casos de otros países emergentes (Nikaido y otros, 2015), aduciendo una toma de decisiones financieras que no cuentan con información suficientes para seleccionar tasas de interés en niveles eficientes que induzcan a una mayor productividad. O incluso la persistencia de fenómenos ligados a brechas de género y localización (Zins \& Weill, 2016).

\section{Conclusiones}

El presente artículo pone de manifiesto la comprensión de la inclusión financiera en países como Colombia, pues en gran medida la política pública y los esfuerzos del gremio financiero se han concentrado en generar un crecimiento en el portafolio de servicios, asumiendo que el hecho instrumental de vincularse y hacer uso de productos tendrá el efecto positivo que la literatura ha compilado frente a la inclusión financiera.

Los coeficientes encontrados en productos como leasing, crédito y seguros, no garantizan por si mismos el aumento en la productividad, pues si los costos transaccionales y las tasas se mantienen en niveles excesivos, erosionan la productividad de los factores, así como amenazan la sostenibilidad de la inclusión. Esta es una situación común en diversos contextos del país, donde las condiciones de educación financiera sumadas a la baja capacidad de negociación de las empresas pequeñas y medianas derivan en una captura de la renta por parte del sector financiero y en un declive en productividad en las empresas.

Las estrategias enfocadas hacia la adopción de un portafolio amplio de productos financieros son insuficientes para generar una dinámica inclusiva en las pequeñas y medianas empresas colombianas, pues los criterios de "uso significativo y sostenibilidad" para los diferentes agentes económicos aún son precarios (Kessler y otros, 2017). A su vez, refuerzan la idea de un mercado que presenta una alta concentración y una limitada eficiencia en términos de costos transaccionales.

Es fundamental que el país avance en estrategias que vinculen regulación y acciones gremiales, encaminadas a fortalecer el uso significativo y la sostenibilidad para avanzar en una real inclusión (Kessler y otros, 2017).

\section{Recomendaciones}

Para trabajos posteriores se recomienda incluir otras variables relativas a la inclusión financiera, por ejemplo, los instrumentos de ahorro que utilizan los establecimientos, el acceso que tienen a los mismos, toda vez que el ahorro se relaciona con la formación de capacidades futuras para la adquisición y mejora de los factores de producción, especialmente a través de la formación bruta de capital fijo, lo que podría relacionarse con variaciones en la productividad. Adicionalmente, se recomienda abordar la inclusión desde los costos transaccionales en comparativos que permitan establecer cuáles serían unos niveles teóricos óptimos.

Desde la política pública al igual que en diversos sectores sería interesante avanzar en una hoja de ruta encaminada a mejorar la inclusión, generando capacidades hacia la adopción amplia y la sostenibilidad de la inclusión. 


\section{Referencias}

[1] Allen, F., Demirguc-Kunt, A., Klapper, L., Martinez Peria, M. S. (2012). The Foundations of Financial Inclusion: Understanding Ownership and Use of Formal Accounts are in the Development. Policy Research Working Paper 6290. The World Bank. http://documents.worldbank.org/curated/en/348241468329061640/The-foundationsof-financial-inclusion-understanding-ownership-and-use-of-formal-accounts. https://doi.org/10.1596/1813-94506290

[2] Anzoategui, D., Demirgüç-Kunt, A., \& Martínez Pería, M. S. (2014). Remittances and Financial Inclusion: Evidence from El Salvador. World Development, 54, 338-349. https://doi.org/10.1016/j.worlddev.2013.10.006

[3] Arias, J. (2016). Sistema Financiero: Un Análisis Del Mercado Desde La Perspectiva Del Derecho Económico. Revista de Derecho Privado, (56), 1-21. https://doi.org/10.5354/0719-7462.2004.41892

[4] Bazhal, I. (2016). The Theory of Economic Development of J.A. Schumpeter: Key Features. MPRA Paper 69883. University Library of Munich. https://mpra.ub.uni-muenchen.de/69883/

[5] Bosma, N., \& Kelley, D. (2018). Global Entrepreneurship Monitor Global Report.

[6] Chauvet, L., \& Jacolin, L. (2017). Financial Inclusion, Bank Concentration, and Firm Performance. World Development, 97, 1-13. https://doi.org/10.1016/j.worlddev.2017.03.018

[7] Chua, J. H., Chrisman, J. J., Kellermanns, F., \& Wu, Z. (2011). Family involvement and new venture debt financing. Journal of Business Venturing, 26(4), 472-488. https://doi.org/10.1016/j.jbusvent.2009.11.002

[8] Cuéllar-Fernández, B., Fuertes-Callén, Y., Serrano-Cinca, C., \& Gutiérrez-Nieto, B. (2015). Determinants of Margin in Microfinance Institutions. Journal Applied Economics (Vol. 48), 300-311. https://doi.org/10.1080/00036846.2015.1078447

[9] Cumming, D., Deloof, M., Manigart, S., \& Wright, M. (2019). New directions in entrepreneurial finance. Journal of Banking \& Finance, 100, 252-260. https://doi.org/10.1016/j.jbankfin.2019.02.008

[10] Daniels, C., Herrington, M., \& Kew, P. (2016). Special Topic Report 2015-2016 Entrepreneurial financing. Global Entrepreneurship Monitor. https://www.gemconsortium.org/report/gem-2015-2016-report-on-entrepreneurialfinancing

[11] Davidsson, P., \& Honig, B. (2003). The role of social and human capital among nascent entrepreneurs. Journal of Business Venturing, 18(3), 301-331. https://doi.org/10.1016/S0883-9026(02)00097-6

[12] De Bettignies, J.-E., Thank, I., Boyer, M., Brander, J., Chemla, G., Clements, M., ... Winter, R. (s. f.). Financing the Entrepreneurial Venture. http://www.sba.gov

[13] Delvasto Perdomo, C. (2006). Asimetrías de información, fallas del mercado crediticio y correctivos institucionales. el papel del estado en la cobertura del mercado crediticio. Criterio Jurídico, 1(6), 215-242. https://revistas.javerianacali.edu.co/index.php/criteriojuridico/issue/view/24 https://doi.org/10.1076/epri.9.9.26.6638

[14] Departamento Administrativo Nacional de Estadísticas -Dane (2018). Información 2018. Encuesta Anual Manufacturera (EAM). https://www.dane.gov.co/index.php/estadisticas-por-tema/industria/encuesta-anualmanufacturera-enam.

[15] Drover, W., Busenitz, L., Matusik, S., Townsend, D., Anglin, A., \& Dushnitsky, G. (2017). A Review and Road Map of Entrepreneurial Equity Financing Research: Venture Capital, Corporate Venture Capital, Angel Investment, Crowdfunding, and Accelerators. Journal of Management, 43(6), 1820-1853. https://doi.org/10.1177/0149206317690584

[16] Eddleston, K. A., Ladge, J. J., Mitteness, C., \& Balachandra, L. (2016). Do You See What I See? Signaling Effects of Gender and Firm Characteristics on Financing Entrepreneurial Ventures. Entrepreneurship: Theory and Practice, 40(3), 489-514. https://doi.org/10.1111/etap.12117

[17] Ferrari, C. (2009). Microfinanzas para el desarrollo. Portafolio. Recuperado de https://search.proquest.com/docview/334476619?accountid=14778 
[18] Fuenzalida, Darcy, \& Mongrut, Samuel. (2010). Estimation of Discount Rates in Latin America: Empirical Evidence and Challenges. Journal of Economics, Finance and Administrative Science, 15(28), 7-43. Recuperado en 09 de agosto de 2020, de http://www.scielo.org.pe/scielo.php?script=sci_arttext\&pid= S2077-18862010000100002\&lng=es\&tlng=en.

[19] Ho, Y.-P., \& Wong, P.-K. (2007). Financing, regulatory costs and entrepreneurial propensity. Small Business Economics, 28(2-3), 187-204. https://doi.org/10.1007/s11187-006-9015-0

[20] Hsu, D. H. (2004). What do entrepreneurs pay for venture capital affiliation? Journal of Finance, 59(4), 18051844. https://doi.org/10.1111/j.1540-6261.2004.00680.x

[21] Isenberg, D. J. (2010). Thew Big Idea. How to Start an Entrepreneurial Revolution. Harvard Business Review.

[22] Karpowicz, I. (2016). Financial Inclusion, Growth and Inequality: A Model Application to Colombia. Journal of Banking and Financial Economics, 2016(2), 68-89. https://doi.org/10.7172/2353-6845.jbfe.2016.2.4

[23] Kerr, W. R., Lerner, J., \& Schoar, A. (2014). The consequences of entrepreneurial finance: Evidence from angel financings. Review of Financial Studies, 27(1), 20-55. https://doi.org/10.1093/rfs/hhr098

[24] Kessler, K., Ikdal, A. S., Naidoo, E., Portafaix, A., Hendrickson, J., Boje, A., \& Rabec, D. (11 de abril, 2017). Improving Financial Inclusion in South Africa. Boston Consulting Group, 20. https://www.bcg.com/publications/2017/globalization-improving-financial-inclusion-south-africa.aspx

[25] Kim, D. W., Yu, J. S., \& Hassan, M. K. (2018). Financial inclusion and economic growth in OIC countries. Research in International Business and Finance, 43, 1-14. https://doi.org/10.1016/j.ribaf.2017.07.178

[26] Klaus Kessler, Adam Ikdal, Euvin Naidoo , Adrien Portafaix , Joshua Hendrickson , Alex Boje, and D. R. (2017). How to Create and Sustain Financial Inclusion. Boston Consulting Group

[27] Kostov, P., Arun, T., \& Annim, S. (2015). Access to financial services: The case of the "Mzansi" account in South Africa. Review of Development Finance, 5(1), 34-42. https://doi.org/10.1016/j.rdf.2015.04.001

[28] Lakshmi, P., \& Visalakshmi, S. (2013). Impact of Cooperatives in Financial Inclusion \& Comprehensive Development. Journal of Finance and Economics, 1(3), 49-53. https://doi.org/10.12691/jfe-1-3-4

[29] Levine, R. (2005). Chapter 12 Finance and Growth: Theory and Evidence. Handbook of Economic Growth, 1, 865-934. https://doi.org/10.1016/S1574-0684(05)01012-9

[30] Levinsohn, J., \& Petrin, A. (2003). Estimating production functions using inputs to control for unobservables. Review of Economic Studies, 70(2), 317-341. https://doi.org/https://doi.org/10.1111/1467-937X.00246

[31] Loufield, E., Ferenzy, D., \& Johnson, T. (2018). Accelerating Financial Inclusion with New Data. Center for Financial Inclusion (CFI) and the Institute of International Finance (IIF). https://content. centerforfinancialinclusion.org/wp-content/uploads/sites/2/2018/08/AcceleratingFINewData_Final_ 2018.06.26.pdf

[32] Maldonado, J. H., \& Tejerina, L. (2010). Investing in large scale financial inclusion: The Case of Colombia. Nota Técnica Banco Inter-Americano de Desarrollo, (IDB-TN-197). Retrieved from http: //publications.iadb.org/bitstream/handle/11319/2750/Investinginlargescalefinancialinclusion: TheCaseofColombia.pdf? sequence $=1$

[33] Manuelito, S., y Jiménez, L. F. (2010). Los mercados financieros en América Latina y el financiamiento de la inversión: hechos estilizados y propuesta para una estrategia de desarrollo. Serie Macroeconomía del Desarrollo, 107. Naciones Unidas. https://repositorio.cepal.org/handle/11362/5337. https://doi.org/10.18356/fd19fa3d-es

[34] Manuelito, S., y Jiménez, L. F. (2013). La inversión y el ahorro en América Latina: nuevos rasgos estilizados, requerimientos para el crecimiento y elementos de una estrategia para fortalecer su financiamiento. Serie Macroeconomía del Desarrollo, 129. Naciones Unidas. https://repositorio.cepal.org/handle/11362/5364. https://doi.org/10.18356/24decf62-es

[35] Mongrut, S., \& Juárez, N. (2018). Valuation of Start-ups: A Behavioral and Strategic Perspective. Revista Mexicana de Economía y Finanzas, 13(3), 419-439. https://doi.org/10.21919/remef.v13i3.314

[36] Nikaido, Y., Pais, J., \& Sarma, M. (2015). What hinders and what enhances small enterprises' access to formal credit in India? Review of Development Finance, 5(1), 43-52. https://doi.org/10.1016/j.rdf.2015.05.002 
[37] Olley, S., \& Pakes, A. (1996). The dynamics of productivity in the telecom- munications equipment industry. Econometrica Vol. 64, No. 6, 1263- 1297. https://doi.org/10.18356/24decf62-es

[38] Ott, T. E., Eisenhardt, K. M., \& Bingham, C. B. (2017). Value Creation through Novel Resource Configurations in a Digitally Strategy Formation in Entrepreneurial Settings: Past Insights and Future Directions. SEJ, 11(3), 195-370. https://doi.org/10.1111/sej.1257

[39] Randøy, T., \& Goel, S. (2003). Ownership structure, founder leadership, and performance in Norwegian SMEs: Implications for financing entrepreneurial opportunities. Journal of Business Venturing, 18(5), 619-637. https://doi.org/10.1016/S0883-9026(03)00013-2

[40] Roa, M., \& Di Giannatale, S. (2016). Formal Saving in Developing Economies: Barriers, Interventions, and Effects. International Development Bank Working Paper Series, 766. https://doi.org/10.18235/0000620

[41] Rojas, J. (2016). Sistema financiero: un análisis del mercado desde la perspectiva del derecho económico. Revista de Derecho Privado, 56, 1-21. https://doi.org/10.18356/24decf62-es

[42] Sarma, M., \& Pais, J. (2010). Financial Inclusion and Development: A Cross Country Analysis. In Annual Conference of the Human Development and Capability Association, New Delhi, 168(10-13), 1-30. https://doi.org/10.1002/jid

[43] Serrao, M., Sequeira, A. H., \& Varambally, K. V. M. (2016). Impact of Financial Inclusion on the Socio-Economic Status of Rural and Urban Households of Vulnerable Sections in Karnataka.

[44] Sharma, D. (2016). Nexus between financial inclusion and economic growth: Evidence from the emerging Indian economy. Journal of Financial Economic Policy, 8(1), 13-36. https://doi.org/10.1108/JFEP-01-2015-0004

[45] Solow, R. (1957). Technical change and the aggregate production function. Review of economics and statistics 39, 312-320. Recuperado el 10 de Agosto, 2020 desde https://www.jstor.org/stable/1926047

[46] Steinert, J. I., Zenker, J., Filipiak, U., Movsisyan, A., Cluver, L. D., \& Shenderovich, Y. (2018). Do saving promotion interventions increase household savings, consumption, and investments in Sub-Saharan Africa? A systematic review and meta-analysis. World Development, 104, 238-256. https://doi.org/10.1016/j.worlddev.2017.11.018

[47] Stiglitz, J., \& Weiss, A. (1981). Credit Rationing in Markets with Imperfect Information. The American Economic Review, 71(3), 393-410. Recuperado agosto 10, 2020, from www.jstor.org/stable/1802787.

[48] Testa, S., Nielsen, K. R., Bogers, M., \& Cincotti, S. (2018). The role of crowdfunding in moving towards a sustainable society. Technological Forecasting and Social Change, 141, 66-73. https://doi.org/10.1016/j.techfore.2018.12.011

[49] Thoene, U., \& Turriago-Hoyos, Á. (2017). Financial inclusion in Colombia: A scoping literature review. Intangible Capital, 13(3), 582-614. https://doi.org/10.3926/ic.946

[50] Tovar, J., Jaramillo, C., \& Hernández, C. (2007). Risk, Concentration and Market Power in Banking Industry, Evidence from the Colombian System (1997-2006). Serie Documentos Cede, 2007-27. Procede. https://core.ac.uk/download/pdf/6325267.pdf. https://doi.org/10.2139/ssrn.1407759

[51] Van Beveren, Ilke, Total Factor Productivity Estimation: A Practical Review (May 24, 2007). LICOS Discussion Paper No. 182/2007, Available at SSRN: https://ssrn.com/abstract=1004429 or https://doi.org/10.2139/ssrn.1004429

[52] Zins, A., \& Weill, L. (2016). The determinants of financial inclusion in Africa. Review of Development Finance, 6(1), 46-57. https://doi.org/10.1016/j.rdf.2016.05.001 